\title{
AN EXTENSIVE LITERATURE SURVEY ON ROUTING PROTOCOLS IN WIRELESS SENSOR NETWORKS
}

Abhishek Soni ${ }^{1}$, Prof. Sandeep Monga ${ }^{2}$

Abstract - The stability and Network lifetime of WSNs depend on sensor nodes power consumption. Optimized the energy consumption, enhance the network lifetime. Mostly Sensor nodes consume energy during the information processing and communication with base station (BS) or other sensor nodes. To extend network lifetime and batter stability, WSNs should be energy efficient. Energy proficiency can be accomplished by various means, as intelligently outlining of MAC and routing protocols. Routing protocols can be flat, Minimum Transmission Energy (MTE) or hierarchical protocols. In In basic routing protocols sensor nodes specifically send information to BS. In MTE every sensor node sends information to its neighbor node; along these lines stack at sensor nodes close to base station is substantially more noteworthy than other sensor nodes, results reduced lifetime. Energy consumption and network life time are fundamental issues in designing of routing protocols for Wireless sensor network. Numerous algorithms have been proposed for diminishing energy utilization and to expand network life time of the WSN. Clustering algorithms have been gained more popularity in this field, as a result of their approach in cluster head detection and information accumulation. Filter (circulated) is the main clustering routing protocol which is turned out to be better contrasted with other such algorithms. This review presents different LEACH protocols for Heterogeneous wireless network.

Indexed Terms - dead nodes; low energy adaptive clustering hierarchy protocol; energy aware multi-hop multi-path hierarchical protocol; heterogeneous wireless sensor networks; energy harvesting.

\section{INTRODUCTION}

With the advancement in smaller scale integrated circuit technology, Wireless Sensor Networks (WSNs) have begun to assume an essential part in our every day lives. It is a direct result of the lessening in cost of the sensor nodes, prompting expanding arrangements of WSNs to a larger degree. Potential applications for wireless sensor networks exist in an assortment of fields, including mechanical process checking and control, condition and living space observing, machine wellbeing checking, home automation, human services applications, atomic reactor control, fire recognition, question following and traffic control. Effective outline and usage of wireless sensor networks have turned into a hot zone of research as of late, because of the huge limit of sensor networks to empower applications associating the physical world with the virtual world. Like living beings, an assortment of current devices and types of gear depends on the sensory information from this present reality around it. These sensory data comes is given by Wireless Sensor Networks (WSN), which includes a couple of minor sensor nodes to screen physical or natural conditions, for instance, temperature, vibration, weight, sound or development, and subsequently aggregately send these information to a central figuring structure, called the base station or sink. Diverse routing protocols administer the development of this data. Broadly the routing protocols can be named level based routing, various leveled based routing, and area based routing. Filter (Low Energy Adaptive Clustering Hierarchy) is a various leveled based routing protocol which utilizes irregular revolution of the nodes required to be the bunch heads to equitably convey energy utilization in the network. Sensor network protocols are very straightforward and consequently are exceptionally helpless to assaults like Sinkhole assault, Selective sending, Sybil assault, Wormholes, HELLO surge assault, Acknowledgment parodying, adjusting, replaying routing data. For instance, Selective sending and HELLO surge assault influences networks with grouping based protocols like LEACH.

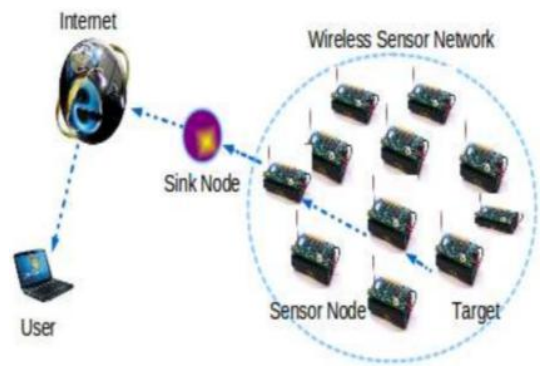

Figure 1.1 Wireless Sensor Network.

\footnotetext{
${ }^{1}$ M.tech Scholar, OIST Bhopal

${ }^{2}$ Research Guide, OIST Bhopal
} 
A wireless sensor network comprises of spatially conveyed self-governing sensors those cooperatively screen the physical or ecological conditions, for example, temperature, sound, vibration, movement, weight or toxins. The WSN is worked of "nodes"- from a couple to a few hundreds or even thousands, where every node is associated with one (or now and again a few) sensors. A structure of a WSN is appeared in Figure 1.1. In sensor networks where nature is should have been remotely checked, the information from the individual sensor nodes is sent to a focal base station (frequently situated a long way from the network), through which the end-client can get to information.

The main characteristics of WSNs include,

- $\quad$ Ease of use

- Ability to cope with node failures

- Communication failures

- Scalability to large scale deployment

- Power consumption constrains for nodes that use batteries or Energy harvesting

- Ability to cooperate with harsh environmental conditions, etc.

\section{THEORY OF LEACH PROTOCOL}

Low Energy Adaptive Clustering Hierarchy (LEACH) is an energy-effective protocol in light of dynamic routing protocol. A different leveled gathering figuring for sensor networks, called Low Energy Adaptive Clustering Hierarchy (LEACH). Filter arranges the nodes in the network into small groups and picks one of them as the bunch head. Node initially faculties its objective and afterward sends the pertinent data to its group head. At that point the bunch head totals and packs the data received from every one of the nodes and sends it to the base station. The nodes picked as the group take deplete off more energy when contrasted with alternate nodes as it is required to send information to the base station which might be far found. Consequently LEACH utilizes arbitrary turn of the nodes required to be the bunch heads to uniformly convey energy utilization in the network.

LEACH operations can be classified in following two phases:-

- Setup phase

- Steady phase

In the setup phase, there are the clusters framed and a cluster head $(\mathrm{CH})$ is decided for every cluster. While in the unfaltering stage, information is detected and sent to the focal base station. The enduring stage is longer than the setup stage. This is done so as to limit the overhead cost.

1. Setup phase: - Amid the setup stage, a foreordained piece of nodes, p, pick themselves as a cluster heads. This is done by a threshold value, T (n). The Threshold regard depends on the desired rate to twist up evidently a cluster head-p, the current round $\mathrm{r}$, and the arrangement of nodes that have not transformed into the gathering head in the last $1 / \mathrm{p}$ rounds, which is demonstrated by G.

2. Steady phase :- During the enduring stage, the sensor nodes i.e. the non-cluster head nodes starts distinguishing data and sends it to their gathering rush toward the TDMA conspire. The gathering head node, ensuing to getting data from all the part nodes, totals it and after that sends it to the base-station.

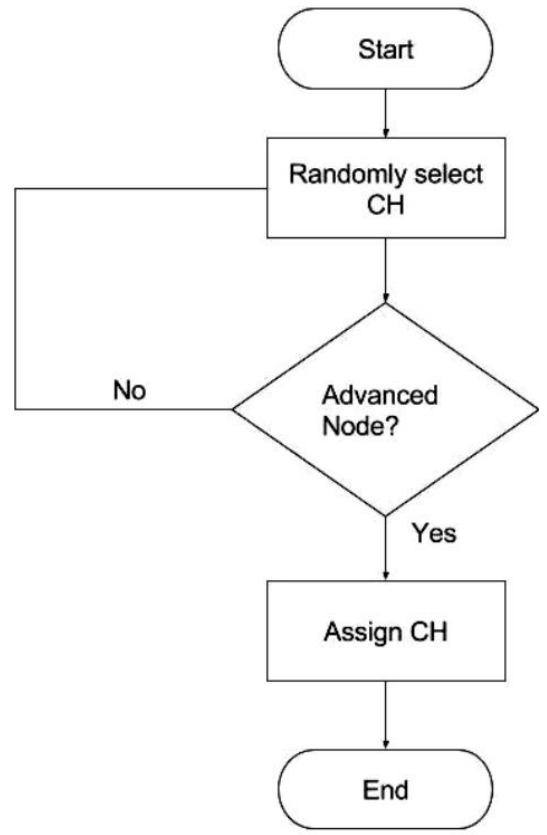

Figure 2.1 Steps in modified LEACH [1]. 
Modified LEACH is isolated into two principle stages at the main setup stage and at the second relentless state stage. In the principal stage nodes are spread arbitrarily allover network region and bunch heads ought to be chosen haphazardly. The choice of group head in the setup period of the changed LEACH is introduced in Fig. 1. As appeared in Fig.1 a CH calculation has been changed to appoint $\mathrm{CH}$ just from cutting edge nodes set. Unfaltering state will continue as before as standard filter endorsed in [8] [9].

\section{RELATED WORK}

\section{A. Amwary, D. Maga and T. Nahdi,[1]}

Wireless sensor networks have strict obliges in size, weight and energy. The conflict of long lifetime and strict energy oblige brings a trademark mix of energy reaping into wireless sensor networks. A balanced low energy adaptable grouping levels of leadership (LEACH) protocol has been proposed to extend the network life time. Modified LEACH is isolated into two guideline stages at the primary setup organize and at the second constant state arrange. Setup organize has been balanced with the ultimate objective that cluster head $(\mathrm{CH})$ decision will be picked just from front line nodes. Results demonstrated basic change in WSN general lifetime. Amusements demonstrate number of dead nodes versus rounds. Connection between's perfect LEACH and balanced one exhibited imperative change in lifetime, similarly examination between adjusted LEACH and energy careful multi-hop multi-path (EAMMH) protocol demonstrates tremendous change in execution.

\section{Kypus, L. Vojtech and J. Hrad, [2]}

The purpose of the Object name services (ONS) stretch out was to find a healthy and stable technique for automation of communication to utilize name and record services to support radio-frequency recognizing (RFID) natural framework, generally in the way that can utilize open source and regulated services and ability to be secured. This work added to the new RFID services and Internet of Things (IoT) heterogeneous conditions capacities presentation. There is a growing enthusiasm of traded data volumes related with each and every IP or non-IP discoverable articles. For example RFID named things and sensors, and furthermore the need to interface remaining communication likeness issues between these two free universes. RFID and IoT environments require delicate execution of security methodologies and techniques. There are as yet noteworthy dangers related with their operations because of the substance nature. One reason of past disappointments could be absence of security as the essential piece of outline of every specific item, which should assemble ONS frameworks. In spite of the fact that it concentrated primarily on the accessibility and classification worries in this exploration, there are still some outstanding zones to be looked into. Attempted to distinguish the solidifying sway by measurements assessing operational status, flexibility, responsiveness and execution of oversaw ONS arrangement plan. Outline of excess and solidified testing condition under tests presented to us the perceivability into the affirmation of the inner communication security and demonstrated conduct under the heap of the segments in such complex data service, as for a general nature of the conveyed ONS service.

\section{A. Girgiri, M. A. Baba, L. G. Ali and M. Adamu,[3]}

Wireless sensor network (WSN) is brilliant innovation that comprises of complex segments, in which low battery life and energy utilization have turned out to be not serious issues. As of now, the utilization of WSN continues expanding, while the issue of energy utilizations additionally increments. A few investigations have been directed to conquer such issues, yet at the same time the issue stays testing. To offer conceivable solutions, there is a need to present new system that manages the issues. This examination proposed conceivable plans for upgrading energy sparing in WSN by enhancing the calculation "Low Energy Adaptive Clustering Hierarchy" (LEACH) Protocol. The examination additionally investigations how to limit energy scattering and power required in WSN contemplating the aggregate power, transmission frequency, transmission separation and transmission time between the sensors in a network. It likewise exhibits energy controls that assistance in comprehending the specified issues in different use of WSN.

\section{P. Shakya, V. Sharma and A. Saroliya,[4]}

Energy consuption is one of the fundamental problem in MANET, productive routing is essential to utilize energy use and enhance the network life time. A mobile Ad hoc network (MANET) is autonomous, self-masterminding and self-planning network with the capacity of speedy sending in view of utilization needs. Channel is an energy build protocol which works in light of the bunch base framework to utilize the energy usage. The bunch arrangement is absolutely depends upon whatever is left of the energy of mobile nodes. The group has persistently having more energy than bunch people. In this examination examinations the execution of LEACH protocol with responsive on ask for multipath AOMDV protocol to profitably utilize the energy constraint in network. Here proposed contrive are not differentiate and any past existing arrangement. The execution of proposed MAX energy based LEACH-AOMDV is differentiated and common LEACH-AOMDV routing and watches that the energy utilize is more in proposed LEACH. In Our examinations exhibit that nodes have, most ideal situation free state information, especially under high traffic rates. Deplete produces group and gives information about energy of each bunch having a place with zone and if energy of the node is higher so LEACH picks that particular node for data transmission that constructs the strong communication. In this examination furthermore explore the result as network parameter like throughput, bundle conveyance proportion, energy utilization by means of node and routing overhead. 
S. H. Gupta, R. K. Singh, J. Bodi and D. Chandhok,[5]

In this exploration expansion to LEACH protocol i.e. Altered LEACH protocol has been systematically and fundamentally broke down. It has been demonstrated that the hard edge an incentive in Modified LEACH protocol directly affects the network life expectancy of a wireless sensor network. Increasingly the underlying energy more will be the net lifetime of the network, as the quantity of alive nodes will be more. Reproduction and systematic outcomes uncover that the Modified LEACH protocol beats the customary LEACH in each perspective.

Zhang Qi and Yu Min, [6]

LEACH based routing protocol is a develop innovation in the earth of minor and static wireless sensor network. In most investigation of mobile WSN, the enhanced LEACH protocol just considered the portability of part nodes. TLEACH protocol proposed in this examination actualized the foundation and support of various leveled topology in a situation that all nodes are mobile. The protocol utilizing tree routing component, control procedure and multi-hop transmit plan to diminish control utilization and enhance conveyance rate. It can be connected to large-scale, dynamical and uneven dispersed mobile WSNs.

Wei Zhang and Liang Zhao, [7]

This examination concentrated on the energy model and calculation depiction of LEACH routing protocol. By breaking down lacking and joining with the genuine circumstance, proposed network model and suppositions for straight structure, the enhanced protocol-clustering join routing protocol LEACH-P that it in view of LEACH and PEGASIS calculation ideological, depicted the network model of LEACH-P protocol which is utilized as a part of the coal confront. This protocol initially picks cluster head in the wireless sensor networks and makes all nodes into clusters. At that point it top-down traversals cluster head and finds a connection association which streamlines cluster head decision and between cluster communication. NS2 reenactment demonstrates that the enhanced protocol can expand the network life cycle and have a decent real-time.

\section{PROBLEM DOMAIN DESCRIPTION}

Introduces that clustering decreases energy utilization in Wireless Sensor Network. LEACH was first clustering protocol. There is couple of protocols in light of LEACH presents with their focal points and weaknesses in this report. Given protocol for homogeneous and furthermore for proactive network and additionally receptive are clarified. Protocol utilized distinctive cluster head decision condition and calculation in which limits number of cluster heads and expands lifetime of network and additionally rest and alert timetable present for expelling energy entire and better dependability period and diverse power levels presented for diminishes energy utilization and decline the retransmission of bundle, crashes and interference for different signals. So energy utilization is lessened and lifetime increments. In responsive protocol delicate edge and hard limit lessens number of transmissions. As indicated by reenactment results and investigation of proposed plans, say that our proposed protocol performs superior to LEACH and MODLEACH. Lifetime of receptive protocol is substantially higher than proactive protocol.

\section{CONCLUSION}

In this research examined the Low Energy Adaptive Clustering Hierarchy (LEACH) protocol and dissected the protocol in light of network lifetime, soundness period and the network throughput. By concentrating light on the correlation of LEAH protocol with the impact of heterogeneity and particular sending assault. Energy effectiveness is despise issue in wireless sensor network (WSN). Various leveled routing or Clustering is best answer for diminishing energy utilization in WSN. Filter (Low energy versatile clustering pecking order) is great various leveled protocol. There are numerous protocols presented in light of LEACH yet at the same time have issue of energy proficiency. Loads of research is going on $\mathrm{CH}$ (cluster head) decision calculation, information total, lessening number of transmission and diverse power levels. Nitty gritty exchange about the current understood protocol for WSNs called LEACH and some of its variations are given. Talked about procedures are a change on it that consolidations the thoughts of various levels in the cluster.

\section{REFERENCES}

[1] A. Amwary, D. Maga and T. Nahdi, "Modified LEACH protocol for heterogeneous wireless networks," 2016 New Trends in Signal Processing (NTSP), Demanovska Dolina, 2016, pp. 1-4.).

[2] L. Kypus, L. Vojtech and J. Hrad, "Security of ONS service for applications of the Internet of Things and their pilot implementation in academic network," Proceedings of the 2015 16th International Carpathian Control Conference (ICCC), Szilvasvarad, 2015, pp. 271-276.)

[3] A. Girgiri, M. A. Baba, L. G. Ali and M. Adamu, "Minimising energy consumption in wireless sensor network by enhancement of leach protocol," 2015 12th International Joint Conference on e-Business and Telecommunications (ICETE), Colmar, 2015, pp. 53-61.)

[4] P. Shakya, V. Sharma and A. Saroliya, "Enhanced multipath LEACH protocol for increasing network life time and minimizing overhead in MANET," 2015 International Conference on Communication Networks (ICCN), Gwalior, 2015, pp. 148-154.)

[5] S. H. Gupta, R. K. Singh, J. Bodi and D. Chandhok, "Evaluation \& analysis of energy efficient extension to LEACH protocol for WSN," 2015 IEEE Power, Communication and Information Technology Conference (PCITC), Bhubaneswar, 2015, pp. 686-690.).

[6] Zhang Qi and Yu Min, "A routing protocol for mobile sensor network based on leach," 10th International Conference on Wireless Communications, Networking and Mobile Computing (WiCOM 2014), Beijing, 2014, pp. 473-477.). 
[7] Wei Zhang and Liang Zhao, "Analysis and research of improved LEACH routing protocol for pressure sensor based on WSN," International Conference on Cyberspace Technology (CCT 2014), Beijing, 2014, pp. 1-6.)

[8] A. Awamry, D. Maga, T. Nahdi, "Energy Consumption of Wirless Sensor Network," in Proceedings of the 17th International Conference on Research in Telecommunication Technologies 2015. Ostrava: VSB TU, 2015, pp. 5-8.

[9] N. Mittal, D. P. Singh, A. Panghal and R. Chauhan, "Improved leach communication protocol for WSN," in National Conference on Computational Instrumentation, 2010, pp. 153-156.

[10] L. Han, "LEACH-HPR: An energy efficient routing algorithm for Heterogeneous WSN," IEEE International Conference on Intelligent Computing and Intelligent Systems (ICIS), 2010, pp. 277-282.

[11] J. Yick, B. Mukherjee and D. Ghosal, "Wireless sensor network survey," Computer networks, 2008, vol. 52, no. 12, pp. $2292-2330$.

[12] I. Akhyildiz and M. C. Vuran, Wirless Sensor Networks, 4 ed., Tsinghua, China: John Wiley \& Sons, 2010. 